\title{
Avaliação da Influência do Regime Térmico em Ambos os Lados da Junta na Soldagem Orbital
}

\author{
Lucas Alves do Nascimento ${ }^{1}$, Louriel Oliveira Vilarinho ${ }^{1}$, Diandro Bailoni Fernandes ${ }^{1}$ \\ ${ }^{1}$ Universidade Federal de Uberlândia, Faculdade de Engenharia Mecânica, Uberlândia, MG, Brasil.
}

Recebido: 20 Jun., 2016

Aceito: 11 Nov., 2016

E-mails: eng.lucasalves@gmail.com (LAN), vilarinho@mecanica.ufu.br (LOV), diandro@mecanica.ufu.br (DBF)
Resumo: O objetivo do presente trabalho é a análise qualitativa da variação de temperatura em ambos os lados da junta soldada, de forma a verificar se esta variabilidade pode ter contribuído no aparecimento de descontinuidades, em destaque a falta de fusão lateral. $O$ aparecimento da descontinuidade pode estar relacionado com a diferença de temperatura em ambos os lados da junta, causada como por exemplo pela quantidade de massa desigual nos dois lados. Para determinar se essas características interferem, foram realizadas soldagens em dutos testes com 3 tubos e 2 tubos, observando-se o regime térmico em pontos simétricos com uso de uma câmera termográfica. As soldagens foram conduzidas em progressão descendente e mecanizada, utilizando-se arame ER70S-6 com 1,2 mm de diâmetro, protegido pela mistura de $\mathrm{Ar}+25 \% \mathrm{CO}_{2}$ com vazão de $15 \mathrm{l} / \mathrm{min}$ em dutos de aço carbono API $5 \mathrm{~L}$ X42 de 8" de diâmetro e $8,0 \mathrm{~mm}$ de espessura. O material foi depositado em junta de topo com chanfro em $\vee$ e bisel de 15, $1 \mathrm{~mm}$ de altura de nariz, $3 \mathrm{~mm}$ de abertura de raiz e sem cobre-junta. Para a junta proposta foi avaliado o processo GMAW derivativo STT (Surface Tension Transfer). Conclui-se que a diferença da temperatura nos lados do duto não afeta a soldagem em geral, sendo a diferença máxima de temperatura entre ambos os lados para pontos simétricos de $4{ }^{\circ} \mathrm{C}$. Já a montagem com dois ou três seções de tubos testes não interferiu significativamente nos resultados.

Palavras-chave: Soldagem de dutos; GMAW; Variação da temperatura; Câmera termográfica; Descontinuidade; Transferência Metálica Controlada.

\section{Analysis Qualitative of the Influence of Flow Thermal on Both Sides of the Joint Welded in Welding Orbital}

\begin{abstract}
This work presents a qualitative analysis of the decrease or increase of the difference temperature in the pipeline through graphics in order to verify if the mass difference and unknown parameters (density etc.) existing on both sides of the installation contributes to the emergence of discontinuities (highlighted the lack of lateral fusion). To determine if these characteristics affect, were carried welds in pipe, observing the thermal flow with use of a thermographic camera in order to analyze the variation of temperature in symmetrical points. The welds were carried out in downhill progression by using orbital displacement device (mechanized welding), ER70S-6 wire with $1.2 \mathrm{~mm}$ diameter and Ar+25\%CO2, as shielding gas (15 l/min). The material was deposited on butt $V$ joint with 15으 bevel, $1 \mathrm{~mm}$ of root face and $3 \mathrm{~mm}$ root opening, without backing. For the proposed joint, GMAW processes with short-circuit metal transfer was assessed the derivative mode by using commercial power source namely STT (Surface Tension Transfer). In this context, decrease and increase temperature was investigated for root pass and filling pass. Were carried out a total of 12 welds ( 6 root pass and 6 filling passes) in two test pipe (with 3 tubes and 2 tubes). After, the recording files ceded by the thermographic camera were decoded and analyzed with of Matlab and OriginPRO ${ }^{\circledR}$ software. The results showed that the rate decrease of temperature the in both sides of the pipe doesn't affect the welding generally because the temperature difference in both sides for symmetrical points in the worst case it's $4{ }^{\circ} \mathrm{C}$.
\end{abstract}

Key-words: Pipe welding; GMAW; Rate temperature; Thermographic camera; Discontinuity; Controlled Metal Transfer. 


\section{Introdução}

$\mathrm{Na}$ análise de transferência de calor, a difusividade térmica $(\alpha)$ mensura a capacidade do material de conduzir energia térmica em relação a sua capacidade de armazená-la, ou seja, expressa quão rapidamente um corpo se ajusta por inteiro à temperatura de seu entorno (no caso, o material na vizinhança da solda). Materiais com valores elevados de difusividade térmica $(\alpha)$ aquecem e resfriam com maior rapidez, isto é, a energia imposta em materiais com difusividade térmica ( $\alpha$ ) muito alta é utilizada em grande parte para aquecer a chapa ou duto teste, dificultando adesão do metal de adição com o metal de base. Assim sendo, a energia imposta no duto deve ser suficiente para fundir o metal de adição, aquecer e fundir o metal de base para aumentar a molhabilidade e evitar a falta de fusão. Conforme apresentado na Equação 1, a difusividade térmica ( $\alpha$ ) é dependente da massa específica, do calor específico e da condutividade térmica dos materiais envolvidos.

$$
\alpha=\frac{k}{\rho \cdot C p}
$$

Onde, " $\rho$ " é a massa específica do material, em kg/m³ e " $\mathrm{Cp}$ " o calor específico, em J/kg.K e " $\mathrm{k}$ " é a condutividade térmica do material, em $\mathrm{W} / \mathrm{mK}$.

Considerando um baixo valor de capacidade térmica volumétrica $(\rho C p)$ para um dado material, significa um tempo menor para o sistema alcançar equilíbrio, ou seja, o sistema tem uma baixa inércia térmica. Desta maneira, para uma mesma energia imposta a variação da massa específica ou material em um dos lados da junta pode contribuir na formação de descontinuidades.

Além disso, para um mesmo conjunto de parâmetros pode-se dizer que o primeiro cordão de solda executado no corpo de prova proposto terá menor probabilidade de apresentar descontinuidades devido à baixa difusividade térmica $(\alpha)$ entre os tubos, uma vez que estarão interligados apenas em determinados pontos por meio de "cachorros ${ }^{1 "}$. Em contrapartida, o quarto cordão de solda (Figura 1), para dutos montados com 3 tubos, poderá ser o mais crítico, ou seja, com maior probabilidade de apresentar descontinuidades, pois, antes da execução deste último cordão de solda os tubos já estarão unidos por outros três cordões de solda, aumentando assim a superfície de contato e consequentemente a rapidez com que o calor se difunde entre os tubos e o ambiente. A mesma análise se aplica ao segundo cordão de solda quando se utilizam 2 tubos testes. Portanto, os cordões de soldas subsequentes ao primeiro poderão necessitar de uma energia de soldagem maior do que ao transmitido no primeiro cordão de solda para evitar uma possível falta de fusão lateral. Neste trabalho será convencionada de duto a união de seções de tubos com 111,5 mm, conforme pode ser observado na Figura 1.

Para determinar se essas características térmicas realmente ocorrem, foram realizadas soldagens em dutos testes, observando-se o regime térmico com uso de uma câmera termográfica, com o intuito de analisar a variação da temperatura em pontos simétricos do corpo de prova. A câmera termográfica recebe radiação do objeto filmado e da vizinhança. Segundo FLIR System (2009) as parcelas de radiações recebidas pela câmera são definidas na Equação 2.

$$
\text { Wtotal }=\varepsilon \tau \text { Wobj }+(1-\varepsilon) \tau W r e f l+(1-\tau) \text { Watm }
$$

Onde, "Wtotal" é a radiação total recebida pela câmera; "Wobj" a emissão de radiação proveniente do objeto; "Wref" a radiação refletida pela superfície do tubo teste; "Watm" a radiação do meio de propagação; " $\varepsilon$ " a emitância do objeto (energia emitida pela superfície de um corpo, por segundo e por unidade de área) e " $\tau$ " a transmitância (fração da luz com um comprimento de onda específico, que atravessa uma determinada matéria).

É notado na Equação 2 que a câmera termográfica recebe uma radiação "Wtotal" à uma temperatura T em curtas distâncias e gera um sinal de saída Ufonte que é linearmente proporcional ao sinal de entrada. Assim sendo, temos a Equação 3.

$$
\text { Wtotal }=\text { CUfonte }
$$

\footnotetext{
1 São pequenos dispositivos com $15 \mathrm{~mm}$ de comprimento, fabricados a partir de cantoneiras de 1"x1/8", posicionados em intervalos regulares internamente aos tubos para garantir que a junta não se altere ao longo da soldagem, sendo 6 unidades por junta. A utilização dos cachorros é para garantir que a junta fique livre de obstruções de forma a não afetar o arco, como poderia ocorrer se fossem utilizados pontos de solda.
} 

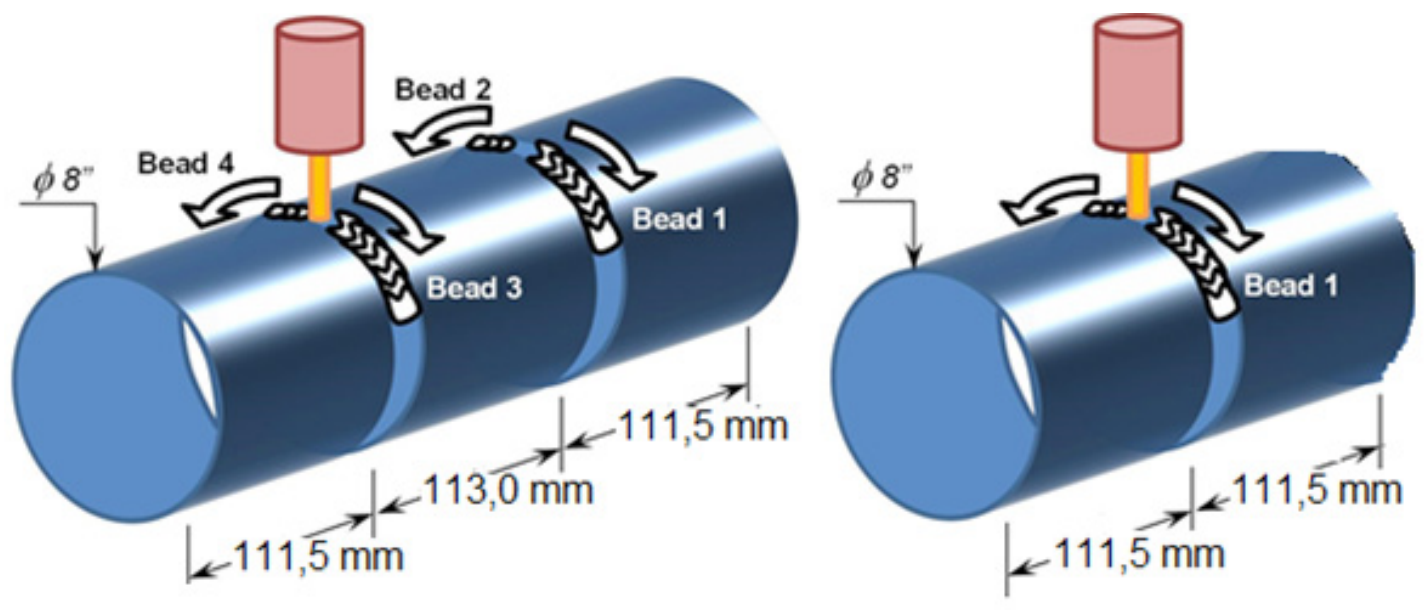

Figura 1. Esquema de execução das soldagens de dutos com 2 e 3 tubos (progressão descendente na posição 5G).

Substituindo a Equação 3 na Equação 2, chega-se na Equação 4.

$$
\text { Utotal }=\varepsilon \tau U o b j+(1-\varepsilon) \tau U r e f l+(1-\tau) U a t m
$$

Assumindo que as tensões monitoradas e teóricas são linearmente proporcionais às temperaturas (T) envolvidas é possível escrever a Equação 5.

$$
\text { Tobj }=\frac{1}{\varepsilon \cdot \tau} \text { Ttotal }-\frac{1-\varepsilon}{\varepsilon} \text { Trefl }-\frac{1-\tau}{\varepsilon \cdot \tau} \text { Tatm }
$$

Dessa forma, analisando-se a Equação 5 é possível determinar a temperatura do objeto conhecendo-se as temperaturas de entrada (convertidas em sinal de tensão ou corrente pela câmera), a emissividade do material e a transmitância do meio. Nota-se também que a radiação refletida do meio é uma variável difícil de controlar, pois, varia com o tempo, ambiente e com o polimento superficial do tubo teste.

A Figura 2 mostra a representação esquemática das variáveis envolvidas na mensuração pela câmera termográfica.

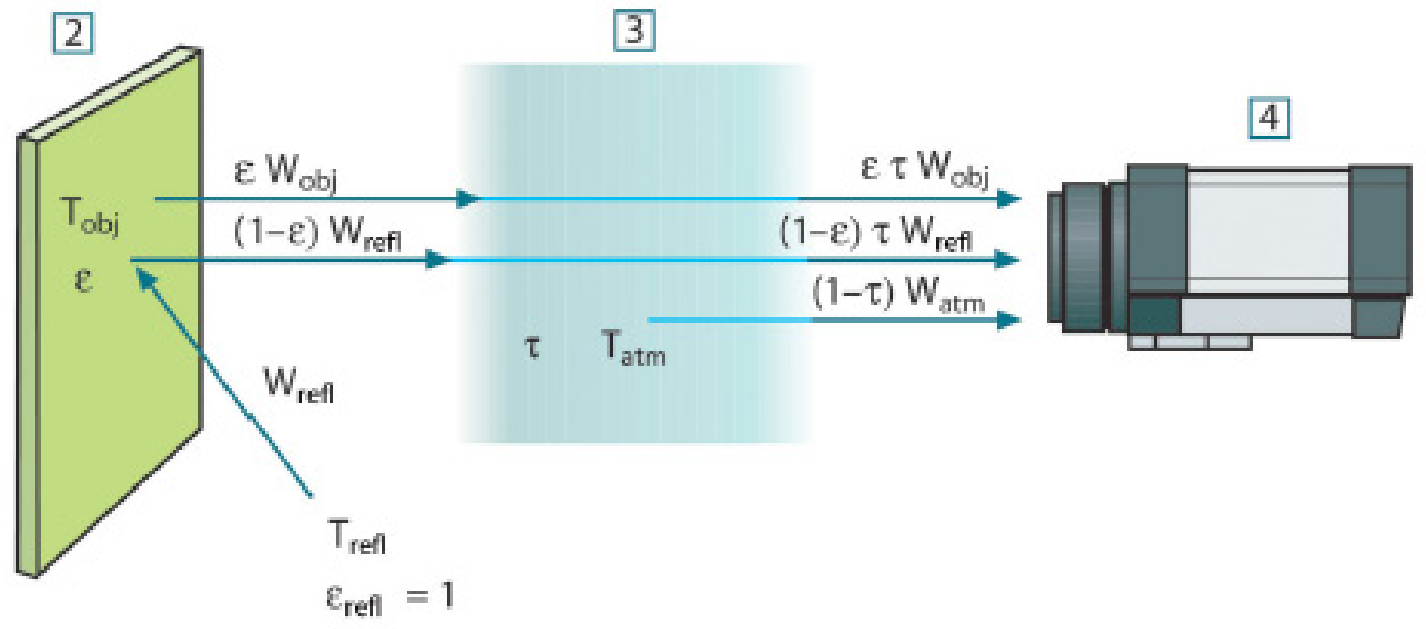

Figura 2. Representação esquemática de medições de temperatura por infravermelho. (1) Vizinhança; (2) Tubo teste/objeto; (3) Atmosfera; (4) Câmera. Adaptado de: FLIR System (2010). 
Dessa forma, este trabalho consiste em analisar qualitativamente a diferença no decaimento ou crescimento de temperatura em pontos simétricos do duto teste (lado tubo e lado suportação) por meio de gráficos, motivado pela elevada frequência de falta de fusão lateral em cordões de soldas presentes em resultados anteriormente investigados pelo Grupo Laprosolda, segundo Vilarinho et al. (2014), em passes de enchimento e de raiz utilizando chanfro estreito e também para garantir que a bancada de testes montada não interfere nas soldagens. Os autores deste trabalho levantam a hipótese de que o aparecimento da descontinuidade pode estar relacionado com a diferença de temperatura em ambos os lados da junta, causada como, por exemplo, pela quantidade de massa desigual nos dois lados da instalação. Destaca-se que os ensaios aqui analisados foram executados nas mesmas condições impostas por Vilarinho et al. (2014). A Figura 3 apresenta exemplos de macrografias obtidas por Vilarinho et al. (2014) utilizando-se o processo derivativo STT.

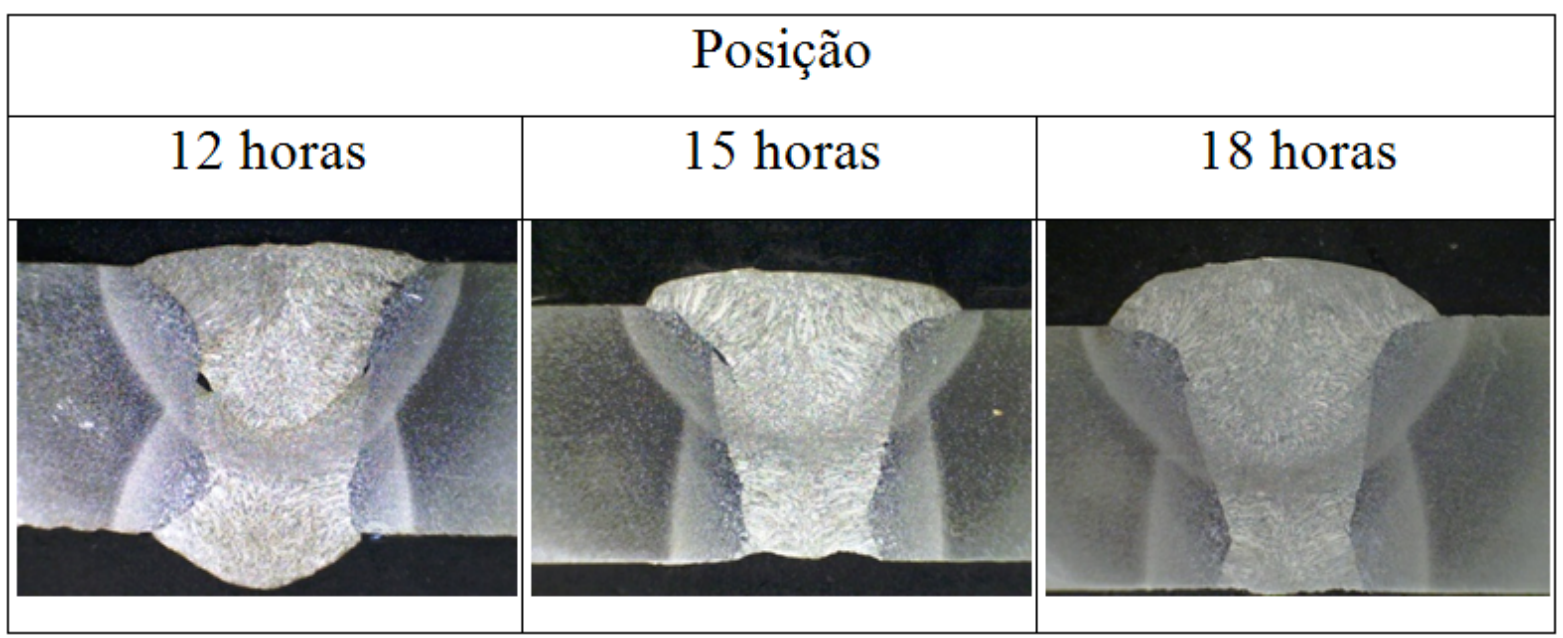

Figura 3. Macrografias dos cordões de solda (espessura da chapa: 8,0 mm). Adaptado de: Vilarinho et al. (2014).

\section{Metodologia}

A bancada experimental montada para a execução dos testes e o posicionamento da câmera termográfica são apresentadas na Figura 4.

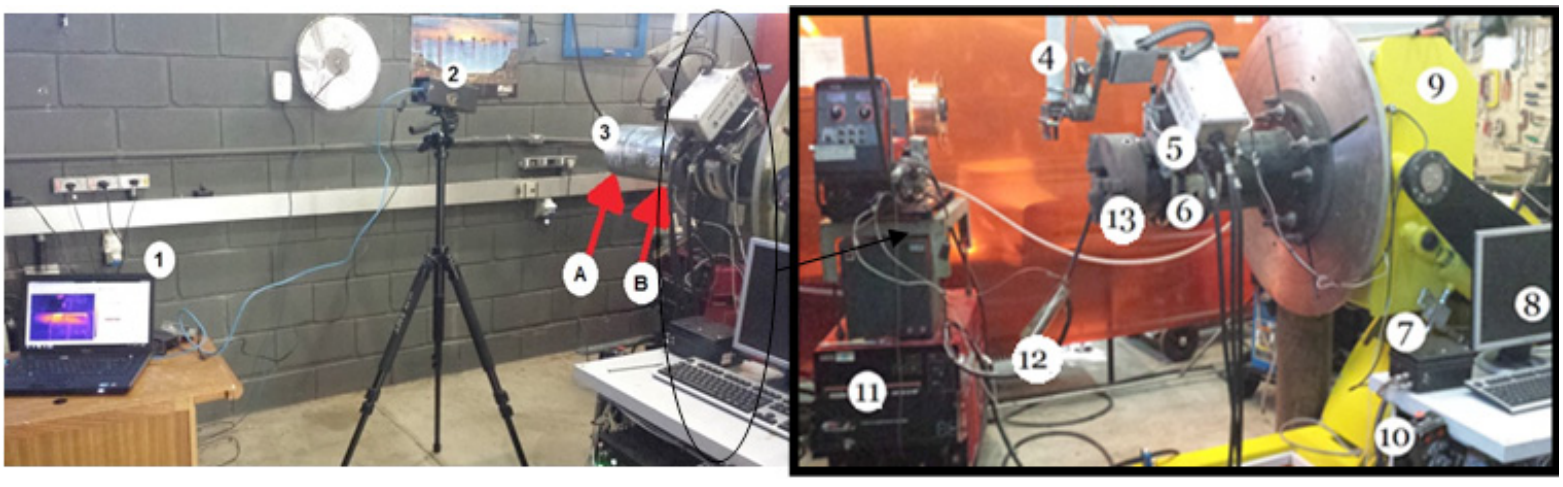

Figura 4. Bancada experimental para filmagem termográfica da soldagem, sendo: 1. Interface de manipulação da câmera termográfica (laptop); 2. Câmera termográfica FLIR A325; 3. Tubo teste; B: Lado suportação e A: Lado do tubo; 4. Tocha de soldagem; 5. Carrinho de movimentação da tocha (Tartílope V4); 6. Cinta de suporte para movimentação angular do carrinho; 7. Sistema de controle de ajuste automático da DBCP; 8. Monitor de visualização dos parâmetros de soldagem e parâmetros de movimento da tocha; 9 . Mesa posicionadora; 10. Controle manual de manipulação da tocha; 11. Fonte de soldagem: Power Wave 450/STT; 12. Cabo terra da fonte de soldagem (aterramento em ambos os lados do tubo); 13. Suporte do duto teste ("castanha"). 
Por convenção, será denominado de "Lado da suportação" o lado do duto teste que é fixo na castanha e "Lado do tubo" o lado que não é suportado. O que separa o "Lado da suportação" do "Lado do tubo" é o chanfro. A partir dessa convenção, é possível observar na Figura 4 que a concentração de massa (aço) é maior em um dos lados da junta ("Lado da suportação"), provocando a perda de calor por condução do tubo teste para a mesa posicionadora, fato que pode contribuir para a falta de fusão lateral. Além do mais a massa específica dos materiais que compõem a mesa posicionadora é desconhecida (lado suportação), existindo a hipótese da difusividade térmica $(\alpha)$ de ambos os lados da junta ser diferente.

Para os ensaios foi utilizado o metal de base cuja análise química é apresentada na Tabela 1. Após a análise química foi realizado ensaio de tração uniaxial no material base (Figura 5) e, comparando estes valores com os valores apresentados na norma API 5L (American Petroleum Institute, 2012), para requisitos mínimos de limites de escoamento e resistência, se pode afirmar que o material analisado aproxima-se do API 5L X42, mesmo aço utilizado por Vilarinho et al. (2014).

Para a execução do processo GMAW (Gas Metal Arc Welding) curto-circuito-controlado STT, utilizou-se fonte de soldagem eletrônica inversora de multiprocessos da fabricante Lincoln Electric de modelo Power Wave 450/STT (Lincoln Electric, 2014).

Os parâmetros utilizados nos testes são apresentados na Tabela 2. O arame eletrodo utilizado foi o AWS ER 70S-6 com diâmetro de 1,2 mm, DBCP (distância bico contato peça) de $12 \mathrm{~mm}$, gás de proteção $\mathrm{Ar}+25 \% \mathrm{CO}_{2}$ com vazão de $15 \mathrm{l} / \mathrm{min}$ e inclinação da tocha em $0^{\circ}$.

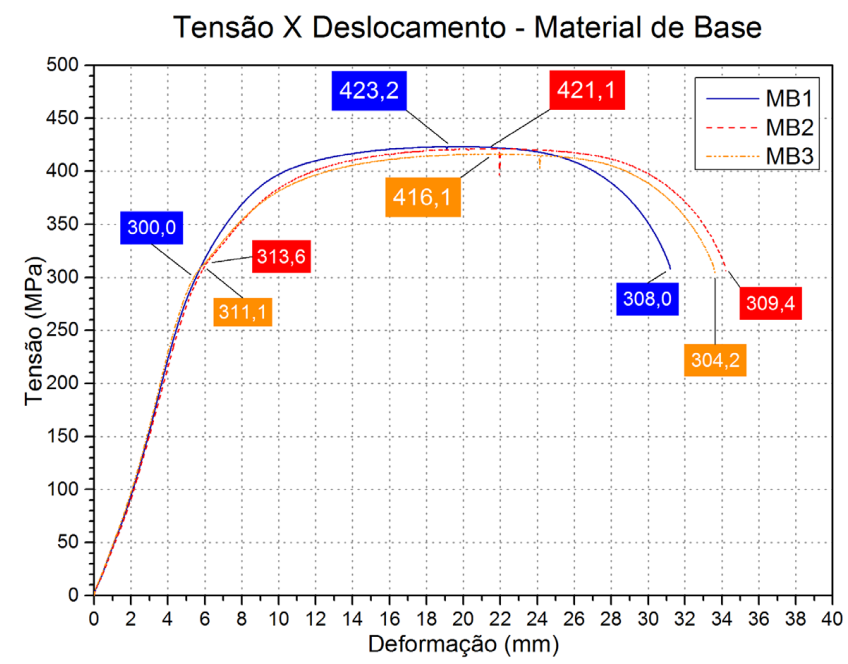

Figura 5. Curvas tensão-deformação para os ensaios de tração uniaxial do metal de base.

Tabela 1. Composição química do metal de base utilizado em porcentagem (\%).

\begin{tabular}{ccccccccc}
\hline $\mathbf{B}$ & $\mathbf{S n}$ & $\mathbf{C a}$ & $\mathbf{C o}$ & $\mathbf{S}$ & $\mathbf{W}$ & $\mathbf{N i}$ & $\mathbf{T i}$ & $\mathbf{C u}$ \\
0,0002 & 0,0012 & 0,0014 & 0,0025 & 0,0033 & 0,0053 & 0,0129 & 0,0171 & 0,0175 \\
\hline $\mathbf{P}$ & $\mathbf{V}$ & $\mathbf{A l}$ & $\mathbf{C}$ & $\mathbf{N b}$ & $\mathbf{S i}$ & $\mathbf{C r}$ & $\mathbf{M o}$ & $\mathbf{M n}$ \\
0,0184 & 0,0253 & 0,031 & 0,0526 & 0,0627 & 0,1866 & 0,2075 & 0,2145 & 1,8165 \\
\hline
\end{tabular}

Tabela 2. Parâmetros do processo derivativo (STT) para ensaios de termografia.

\begin{tabular}{|c|c|c|c|c|c|c|c|c|}
\hline Passe & $\begin{array}{c}\text { Va* } \\
(\mathrm{m} / \mathrm{min})\end{array}$ & $\begin{array}{c}V_{s}^{*} \\
(\mathrm{~cm} / \mathrm{min})\end{array}$ & $\begin{array}{l}\text { Amplitude de } \\
\text { tecimento (mm) }\end{array}$ & $\begin{array}{l}\text { Frequência de } \\
\text { tecimento }(\mathrm{Hz})\end{array}$ & $\begin{array}{l}\mathrm{Ib}^{*} \\
(\mathrm{~A})\end{array}$ & $\begin{array}{l}\text { Ip* } \\
\text { (A) }\end{array}$ & Tout* & $\begin{array}{c}\text { Burnback } \\
\text { time(s)* }\end{array}$ \\
\hline Raiz & 5,75 & $35^{* *}$ & 3 & 3,6 & 130 & 350 & 10 & 0,03 \\
\hline Enchimento & 4,20 & $22,7^{* *}$ & 5 & 2,4 & 130 & 330 & 8 & 0,03 \\
\hline
\end{tabular}

*Va: Velocidade de alimentação do arame; Vs: Velocidade de soldagem; Ib: Corrente de base; Ip: Corrente de pico; Tout: Tail-out - em analogia ao processo por curto-circuito convencional este parâmetro é equivalente a uma regulagem de indutância; Burnback time: parâmetro que evita o arame colar no cordão de solda ao término do processo. **A velocidade de soldagem parametrizada no console do Tartilope é dada por Vs*1,277. 
As soldagens foram conduzidas de forma mecanizada utilizando o manipulador de tocha da marca Tartilope V4, executadas em progressão descendente e em dois passes (um de raiz e outro de enchimento/acabamento). Como forma de facilitar a execução de soldagens de campo, cada junta soldada foi executada com o mesmo processo/fonte, ou seja, tanto a raiz, quanto o enchimento foram feitos com este processo e, ambas com transferência por curto-circuito.

Foram realizados um total de 12 cordões de solda ( 6 passes de raiz e 6 passes de enchimento) em dois tubos testes, sendo um deles montados com 3 tubos e outro com 2 tubos. A realização dos passes em progressão descendente foram feitas em duas etapas. A primeira começa no ponto mais alto do tubo, às 12 horas $\left(0^{\circ}\right)$, passando pela posição vertical às 15 horas $\left(90^{\circ}\right)$ e termina no ponto mais baixo, às 18 horas $\left(180^{\circ}\right)$, por um lado. A segunda etapa do processo começa novamente às 12 horas e o cordão é depositado em volta do outro lado do tubo até se encontrar com o primeiro cordão, às 18 horas (Figura 6).

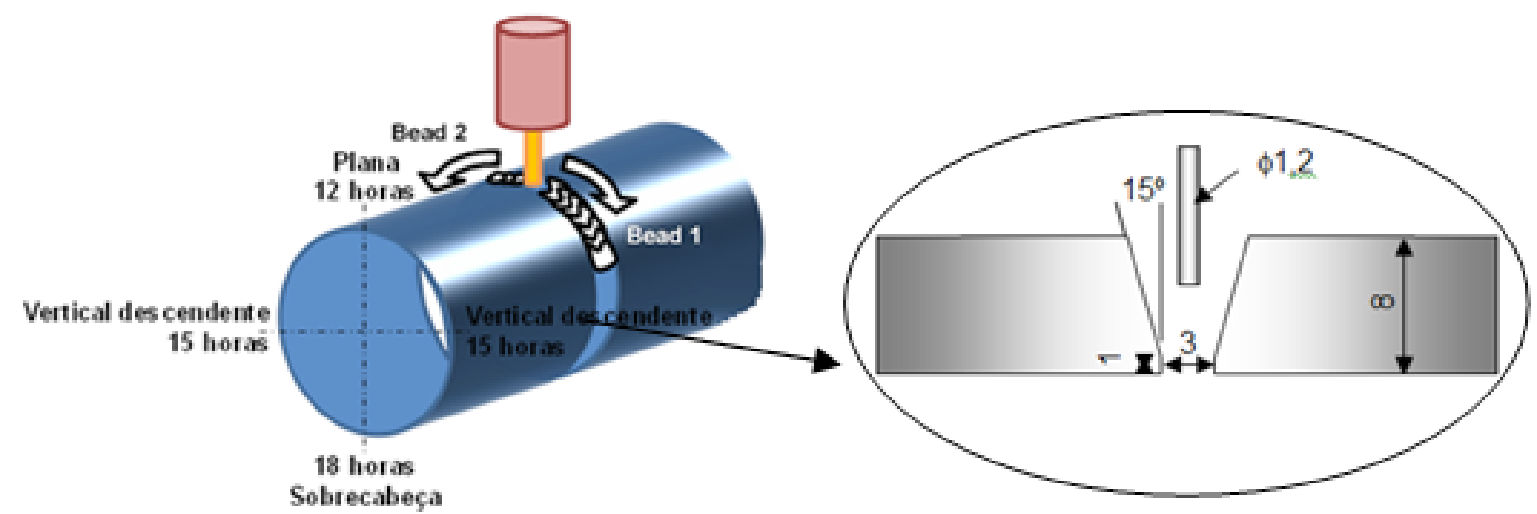

Figura 6. Junta de topo com preparação em $\vee$ utilizada mostrando o arame-eletrodo como comparativo esquemático (escala 2:1 e cotas em $\mathrm{mm}$ ).

Posteriormente, os arquivos de gravação cedidos pela câmera termográfica de modelo FLIR A325 foram decodificados e analisados pelo software Matlab ${ }^{\circledR}$ e, com o auxílio do software OriginPRO ${ }^{\circledR}$ foram gerados os gráficos. Já as soldagens foram instrumentadas, em conformidade com a ISO 17662 (International Organization for Standardization, 2005), com sistema de aquisição de sinais de corrente e tensão desenvolvido por Machado (2011), onde os valores obtidos para cada um dos passes foram também tratados pelo software OriginPRO ${ }^{\circledR}$.

\subsection{Ajuste da câmera termográfica}

Com base na tabela de emissividade exibida no manual da câmera (FLIR System, 2009) adotou-se a emissividade de 0,760 (ferro em temperatura até $538^{\circ} \mathrm{C}$ ). Para a transmitância foi selecionada o valor padrão igual a 1. A temperatura de saturação da câmera durante as filmagens foi fixada em $510{ }^{\circ} \mathrm{C}$. Todos os parâmetros empregados no experimento são apresentados na Tabela 3.

Segundo Mota (2011), as imagens filmadas devem ser corrigidas conforme a inclinação da câmera fotográfica de modo a não interferir nos resultados finais, ou seja, quanto maior o ângulo de inclinação, maior será a distorção observada na imagem adquirida. Neste trabalho em particular, o eixo óptico da câmera foi ajustado, de modo a focar diretamente para a linha de interesse (descendente vertical do duto teste) tornando as distorções nulas. Neste caso não foi necessário planificar ou retificar a imagem.

Tabela 3. Parametrização da câmera termográfica.

\begin{tabular}{ccccc}
\hline Emissividade & $\begin{array}{c}\text { Distância entre tubo } \\
\text { teste e câmera }(\mathbf{m})\end{array}$ & $\begin{array}{c}\text { Temperatura da radiação } \\
\text { refletida (Trefl.) em }{ }^{\circ} \mathbf{C}\end{array}$ & $\begin{array}{c}\text { Temperatura devido a } \\
\text { radiação do ambiente } \\
\text { (Tatm) em }{ }^{\circ} \mathbf{C}\end{array}$ & Transmitância \\
\hline 0,760 & 0,85 & 25 & 33 & 1 \\
\hline
\end{tabular}


A linha de interesse a ser analisada foi selecionada de acordo com as limitações físicas na fixação da câmera e em virtude das descontinuidades ocorrerem na posição vertical. Tais linhas de interesse são ilustradas na Figura 7 e as coordenadas consideradas no estudo são mostradas na Tabela 4.
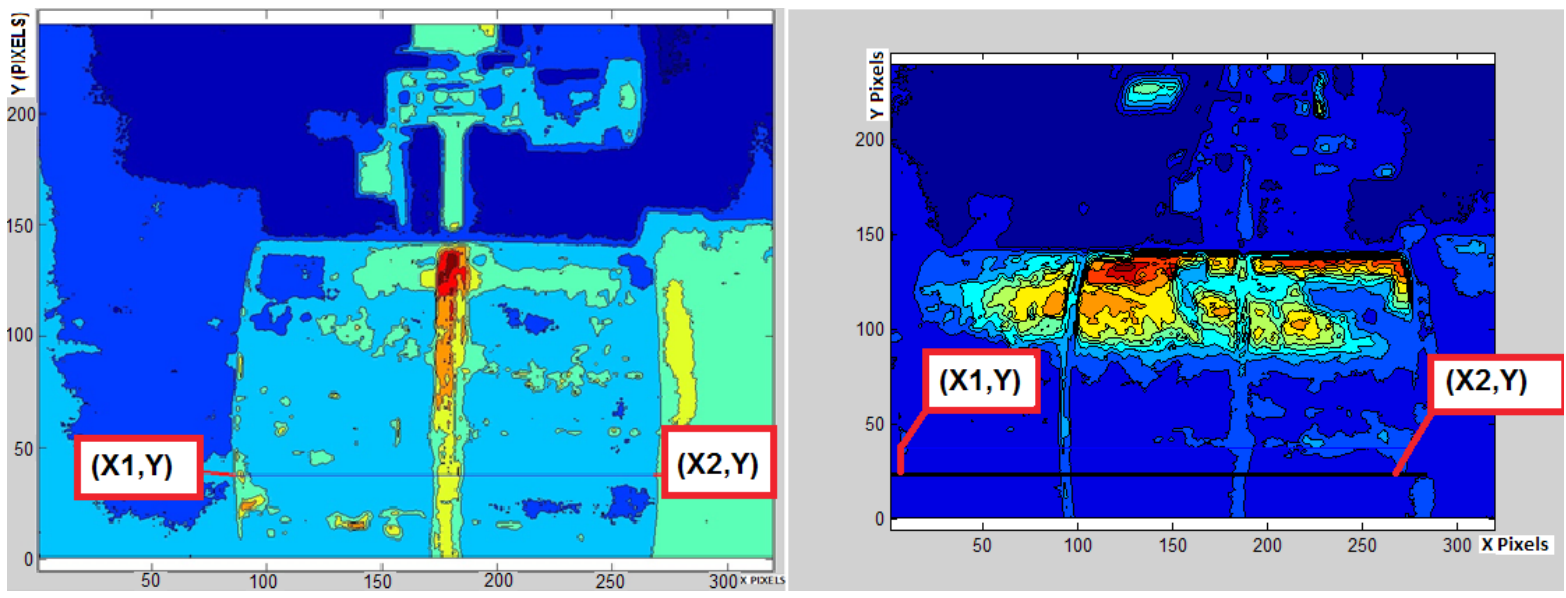

Figura 7. Linha de interesse utilizada na imagem térmica.

Tabela 4. Coordenadas em pixels das linhas de referência utilizada no ensaio termográfico.

\begin{tabular}{ccc}
\hline Posições & 2 Tubos & 3 Tubos \\
X1 & 85 & 5 \\
X2 & 269 & 275 \\
Y & 38 & 20 \\
\hline
\end{tabular}

\subsection{Seleção dos pontos simétricos}

Para auxiliar na seleção dos pontos simétricos foram implementados códigos em Matlab ${ }^{\circledR}$ para decodificação das imagens em matrizes de dados numéricos, geração de filmes em 3D e geração de gráficos em função do tempo cuja coordenada da linha analisada possa ser modificada com flexibilidade.

Após a fixação das linhas que serão analisadas (Tabela 4), o primeiro passo da análise é expurgar os quadros iniciais que representam a passagem da tocha na frente do cordão de solda. A passagem da tocha que se localiza entre o cordão de solda e a câmera causam desvios significativos nos dados mensurados. Tal fato é percebido observando-se a desestabilização em sequência dos gráficos disponibilizados pelo software Matlab ${ }^{\circledR}$ durante a movimentação da tocha.

Com o auxílio do filme em 3D, decodificado pelo Matlab ${ }^{\circledR}$, em sincronismo com gráficos gerados foi possível identificar os quadros iniciais da filmagem que devem ser expurgados (Figura 8). Neste contexto, durante a passagem da tocha em frente à câmera termográfica os gráficos ficam distorcidos (Figura 8a e b) até o momento em que o gráfico se transforma em uma gaussiana (Figura 8d). O momento ideal para o inicio das análises qualitativas é marcado ao ser observado a formação da curva gaussiana e, em vista disso todos os dados anteriores a 5000 quadros foram expurgados.

Na prática alguns pontos adquiridos pela câmera termográfica foram somados as radiações térmicas refletidas, ocasionada pela incidência da luz ambiente sobre a superfície (componentes de radiação $T_{\text {refl }}$ e $\varepsilon_{\text {refl }}$ mostrada na Figura 2). Confrontando o gráfico e a imagem térmica de um dos ensaios representados pela Figura 9a, b pode-se dizer que do ponto 200 ao 275 existe uma refletância elevada provocada pelo acabamento superficial. Portanto, para análise da soldagem com 3 tubos foi necessário modificar a coordenada " $Y$ " da linha de referência de 38 pixels para 20 pixels, devido à presença desta refletância na soldagem do passe de enchimento.

Na Figura 10 percebe-se também que a temperatura anterior ao ponto 85 é menor, pois, os tubos estão unidos apenas por "cachorros", o que diminui a eficiência de condução do calor para a outra parte do tubo. A fita 


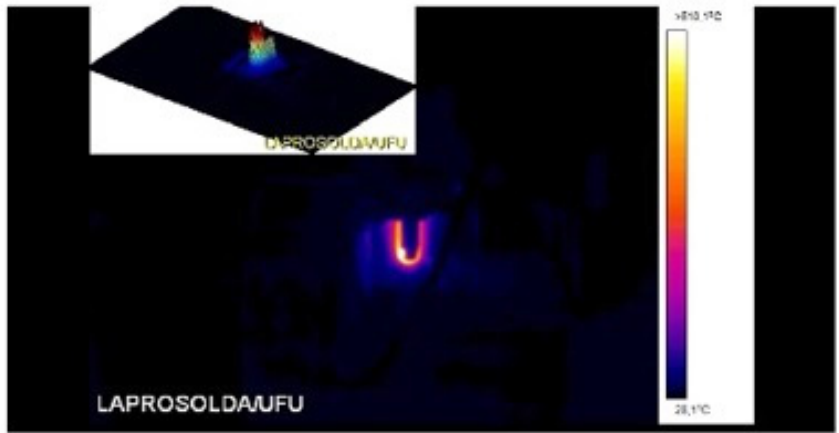

(a)

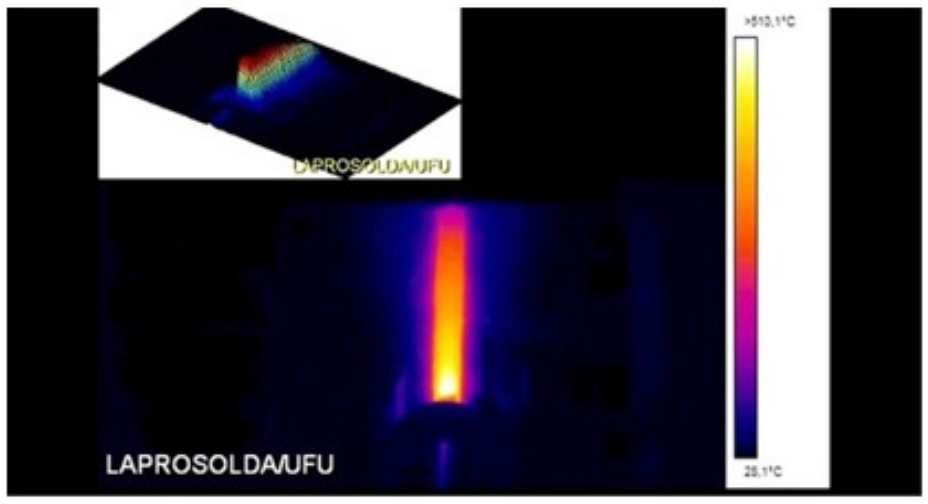

(c)

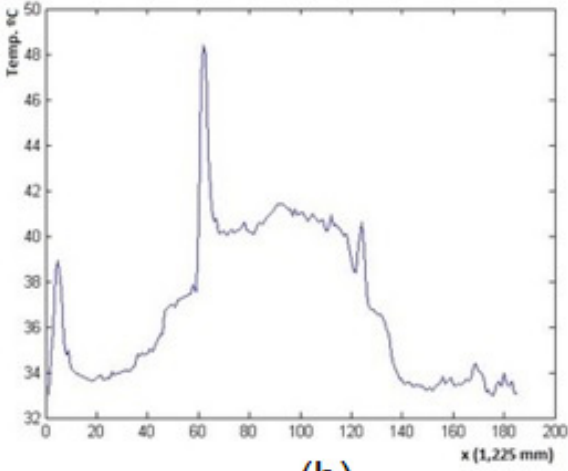

(b)

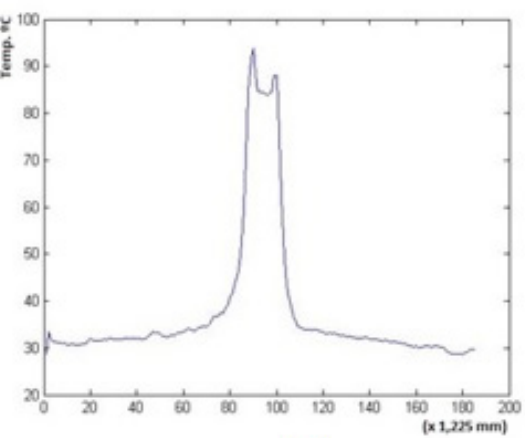

(d)

Figura 8. Sincronismo entre filmagem e gráficos gerados no software Matlab ${ }^{\circledR}$ : (a) Imagem do filme em 3D no instante da passagem da tocha em frente à câmera; (b) gráfico representando a imagem mostrada em (a); (c) Imagem do filme em 3D no instante após a passagem da tocha na frente à câmera; (d) gráfico representando a imagem mostrada em (c).

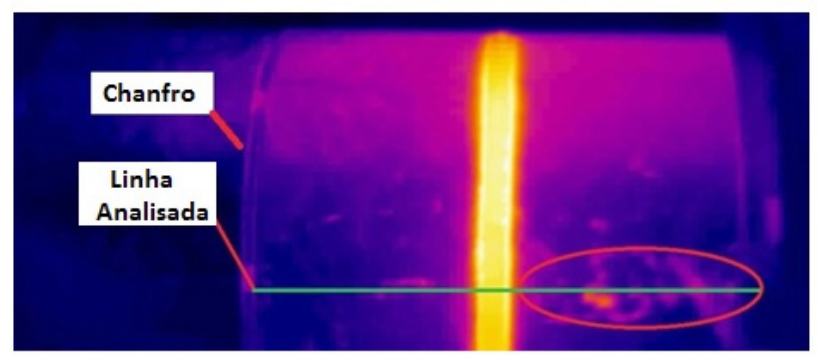

(a)

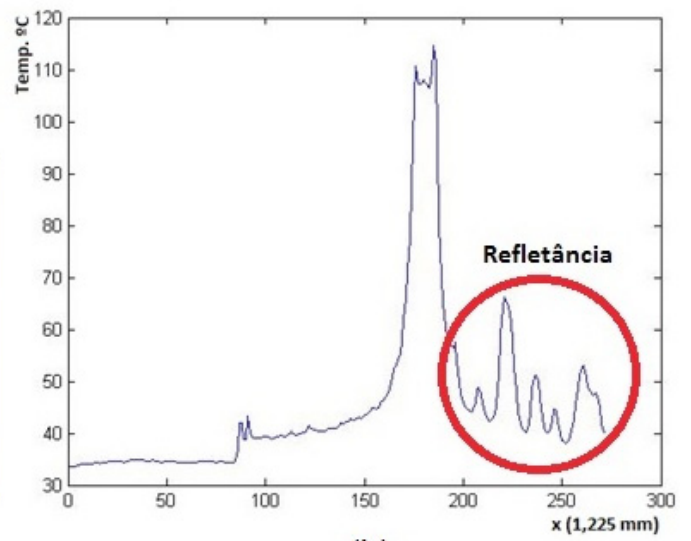

(b)

Figura 9. Imagem justificando a expurga dos dados para a refletância provocada pelo acabamento superficial; (a) Ensaio 3T_E1; e (b) gráfico correspondente.

branca, utilizada para auxiliar no controle automático da DBCP, é facilmente identificada nos gráficos de passe de raiz, assim como a presença do chanfro entre os pontos 85 e 96.

Todos os pontos que apresentam as anomalias acima foram descartados em todos os ensaios realizados, pois, variam desordenadamente e não representam a variação de temperatura no duto. 


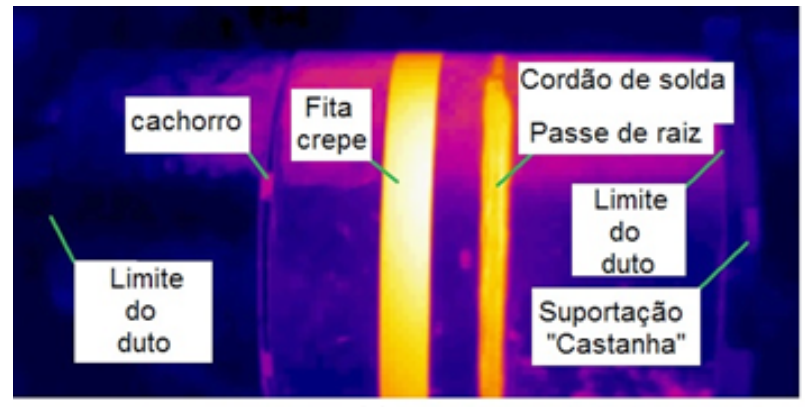

(a)

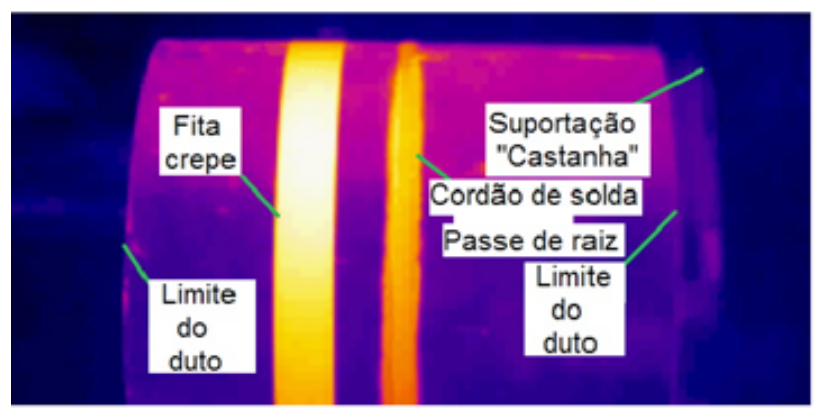

(c)

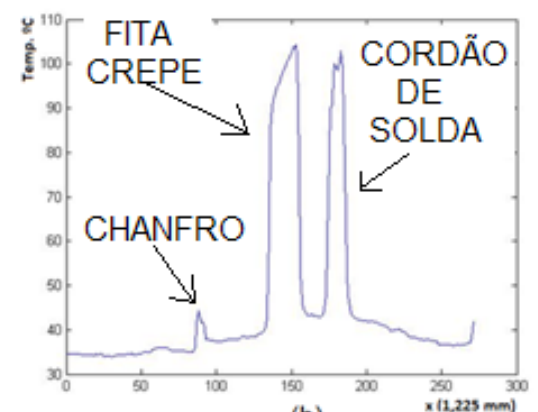

(b)

FITA

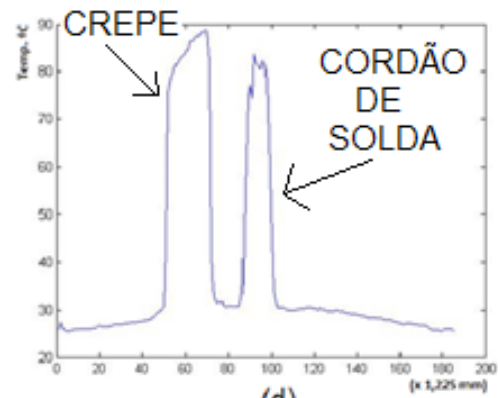

(d)

Figura 10. Imagens térmicas e respectivos gráficos processados em Matlab ${ }^{\circledR}$ : (a) ensaio 3T_R1 e (b) seu gráfico correspondente; (c) ensaio 2T_R2; e (d) seu gráfico correspondente.

Posteriormente ao procedimento de expurga dos dados são selecionados os pontos para análise do decaimento ou crescimento da temperatura. $O$ critério de escolha destes pontos foi baseado na observação de todos os pontos do perfil de temperatura em função do tempo, nas linhas referências fixadas na Tabela 4, durante toda a execução da soldagem da meia cana e no período de resfriamento e, selecionado os pontos simétricos (em relação ao centro do chanfro) que não apresentaram distorções. A Tabela 5 discrimina todos os ensaios e pontos simétricos selecionados.

\section{Resultados}

A partir dos parâmetros de soldagem definidos na Tabela 2, realizaram-se as soldagens de forma a verificar os valores de corrente e tensões próximas ao trabalho de Vilarinho et al. (2014) e também para corroborar com as características do processo derivativo (STT) de soldagem utilizado. Como resultados, são apresentados na Figura 11,
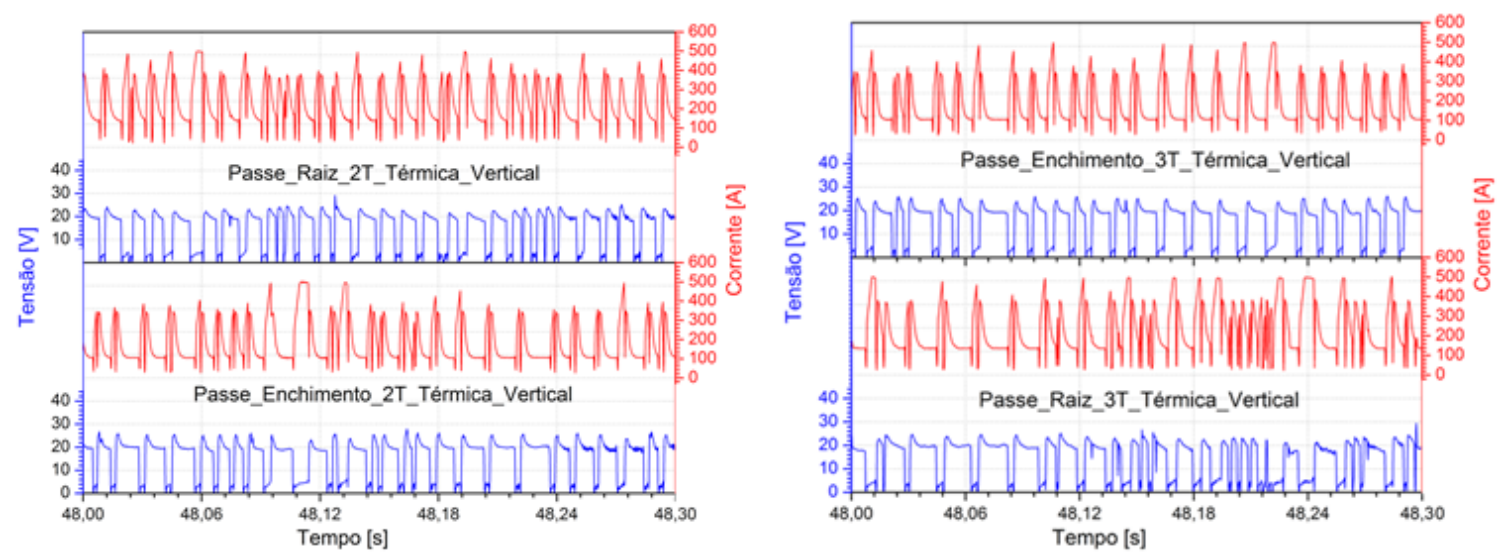

Figura 11. Exemplo de oscilogramas reais adquiridos para passes de raiz e enchimento utilizando o processo derivativo (STT). 
Tabela 5. Pontos selecionados para análise do decaimento ou crescimento da temperatura.

\begin{tabular}{|c|c|c|c|c|c|c|}
\hline Ensaio & $\begin{array}{l}\text { Início } \\
\text { (quadro) }\end{array}$ & $\underset{\text { (quadro) }}{\text { Fim }}$ & $\begin{array}{l}\text { Número total de } \\
\text { quadros gravados }\end{array}$ & $\begin{array}{l}\text { Ponto central da } \\
\text { largura cordão } \\
\text { (pixels) }\end{array}$ & $\begin{array}{l}\text { Pontos } \\
\text { simétricos } \\
\text { (pixels) }\end{array}$ & $\begin{array}{c}\text { Coordenadas dos } \\
\text { pontos analisados } \\
\text { em relação ao ponto } \\
\text { central ( } \mathrm{mm})\end{array}$ \\
\hline 2T_E1 & 5000 & 11000 & 18861 & 94 & $\begin{array}{l}(74,114) ; \\
(54,134) ; \\
(26 ; 162)\end{array}$ & $\begin{array}{c}0,0 \\
(-24,5 ; 24,5) \\
(-49 ; 49) \\
(-83,3 ; 83,3)\end{array}$ \\
\hline 2T_R1 & 5000 & 11000 & 18478 & 94 & $\begin{array}{l}(42,146) ; \\
(22,166)\end{array}$ & $\begin{array}{c}0,0 \\
(-63,7 ; 63,7) \\
(-88,2 ; 88,2) \\
\end{array}$ \\
\hline 2T_E2 & 5000 & 11000 & 18026 & 94 & $\begin{array}{l}(74,114) \\
(54,134) \\
(26 ; 162)\end{array}$ & $\begin{array}{l}\quad 0,0 \\
(-24,5 ; 24,5) \\
(-49 ; 49) \\
(-83,3 ; 83,3)\end{array}$ \\
\hline 2T_R2 & 5000 & 11000 & 18110 & 94 & $\begin{array}{l}(42,146) \\
(22,166)\end{array}$ & $\begin{array}{c}0,0 \\
(-63,7 ; 63,7) \\
(-88,2 ; 88,2) \\
\end{array}$ \\
\hline 3T_E1 & 5000 & 11000 & 18300 & 180 & $\begin{array}{l}(160,200) ; \\
(140,220) ; \\
(110,250)\end{array}$ & $\begin{array}{l}\quad 0,0 \\
(-24,5 ; 24,5) \\
(-49 ; 49) \\
(-85,75 ; 85,75) \\
\end{array}$ \\
\hline 3T_R1 & 5000 & 11000 & 16083 & 190 & $\begin{array}{l}(175,205) ; \\
(130,250) ; \\
(115,265)\end{array}$ & $\begin{array}{c}0,0 \\
(-18,4 ; 18,4) \\
(-73,5 ; 73,5) \\
(-91,9 ; 91,9) \\
\end{array}$ \\
\hline 3T_E2 & 5000 & 11000 & 18728 & 174 & $\begin{array}{l}(154,194) ; \\
(134,214) ; \\
(98,250) ;\end{array}$ & $\begin{array}{l}0,0 \\
(-24,5 ; 24,5) \\
(-49 ; 49) \\
(-93,1 ; 93,1) \\
\end{array}$ \\
\hline 3T_R2 & 5000 & 11000 & 18183 & 180 & $\begin{array}{l}(165,195) ; \\
(120,240) ; \\
(100,260)\end{array}$ & $\begin{array}{c}0,0 \\
(-18,4 ; 18,4) \\
(-73,5 ; 73,5) \\
(-98,0 ; 98,0) \\
\end{array}$ \\
\hline 3T_E3 & 5000 & 11000 & 18667 & 175 & $\begin{array}{l}(155,195) ; \\
(135,215) ; \\
(105,245) ;\end{array}$ & $\begin{array}{l}\quad 0,0 \\
(-24,5 ; 24,5) \\
(-49 ; 49) \\
(-85,8 ; 85,8) \\
\end{array}$ \\
\hline 3T_R3 & 5000 & 11000 & 18386 & 175 & $\begin{array}{l}(160,190) ; \\
(115,235) ; \\
(100,250)\end{array}$ & $\begin{array}{c}0,0 \\
(-18,4 ; 18,4) \\
(-73,5 ; 73,5) \\
(-91,9 ; 91,9) \\
\end{array}$ \\
\hline 3T_E4 & 5000 & 11000 & 18488 & 175 & $\begin{array}{l}(155,195) ; \\
(135,215) ; \\
(105,245) ;\end{array}$ & $\begin{array}{l}\quad 0,0 \\
(-24,5 ; 24,5) \\
(-49 ; 49) \\
(-85,8 ; 85,8) \\
\end{array}$ \\
\hline 3T_R4 & 5000 & 11000 & 18182 & 175 & $\begin{array}{l}(160,190) ; \\
(115,235) ; \\
(100,250)\end{array}$ & $\begin{array}{c}0,0 \\
(-18,4 ; 18,4) \\
(-73,5 ; 73,5) \\
(-91,9 ; 91,9) \\
\end{array}$ \\
\hline
\end{tabular}

A identificação dos cordões de solda segue o seguinte código: NT_(R ou E)X, onde NT: Número de tubos, R: passe de raiz, E: passe de enchimento e $X$ número do ensaio. Ex.: 3T_R1 (ensaios 1 para passe de raiz com 3 tubos).

os oscilogramas típicos, e na Tabela 6, os valores típicos de corrente e tensão adquiridos durante as soldagens. Os dados exibidos na Tabela 6 foram calculados usando o programa desenvolvido em Matlab ${ }^{\circledR}$.

De forma geral, os oscilogramas obtidos aproximam-se do gráfico conseguido por Vilarinho et al. (2014) para o processo de soldagem derivativo (STT). Os resultados apresentados na Tabela 1 são satisfatórios para a análise 
Tabela 6. Valores típicos de corrente e tensão adquiridos durante a avaliação termográfica.

\begin{tabular}{lcccc}
\hline \multicolumn{1}{c}{ Teste } & Corrente Média [A] & Corrente RMS [A] & Tensão Média [V] & Tensão RMS [V] \\
2T_Enchimento & 197 & 228 & 15,2 & 17,4 \\
2T_Raiz & 239 & 266 & 14,6 & 16,9 \\
3T_Enchimento & 200 & 232 & 15,0 & 17,2 \\
3T_Raiz & 227 & 254 & 15,3 & 17,5 \\
\hline
\end{tabular}

termográfica, pois segundo Vilarinho et al. (2014) a corrente média deveria ser maior que 173A para o passe de enchimento e maior ou igual a 220 A para o passe de raiz para evitar uma possível falta de fusão. Na Figura 12 são mostrados exemplos de cordões de solda fabricados.
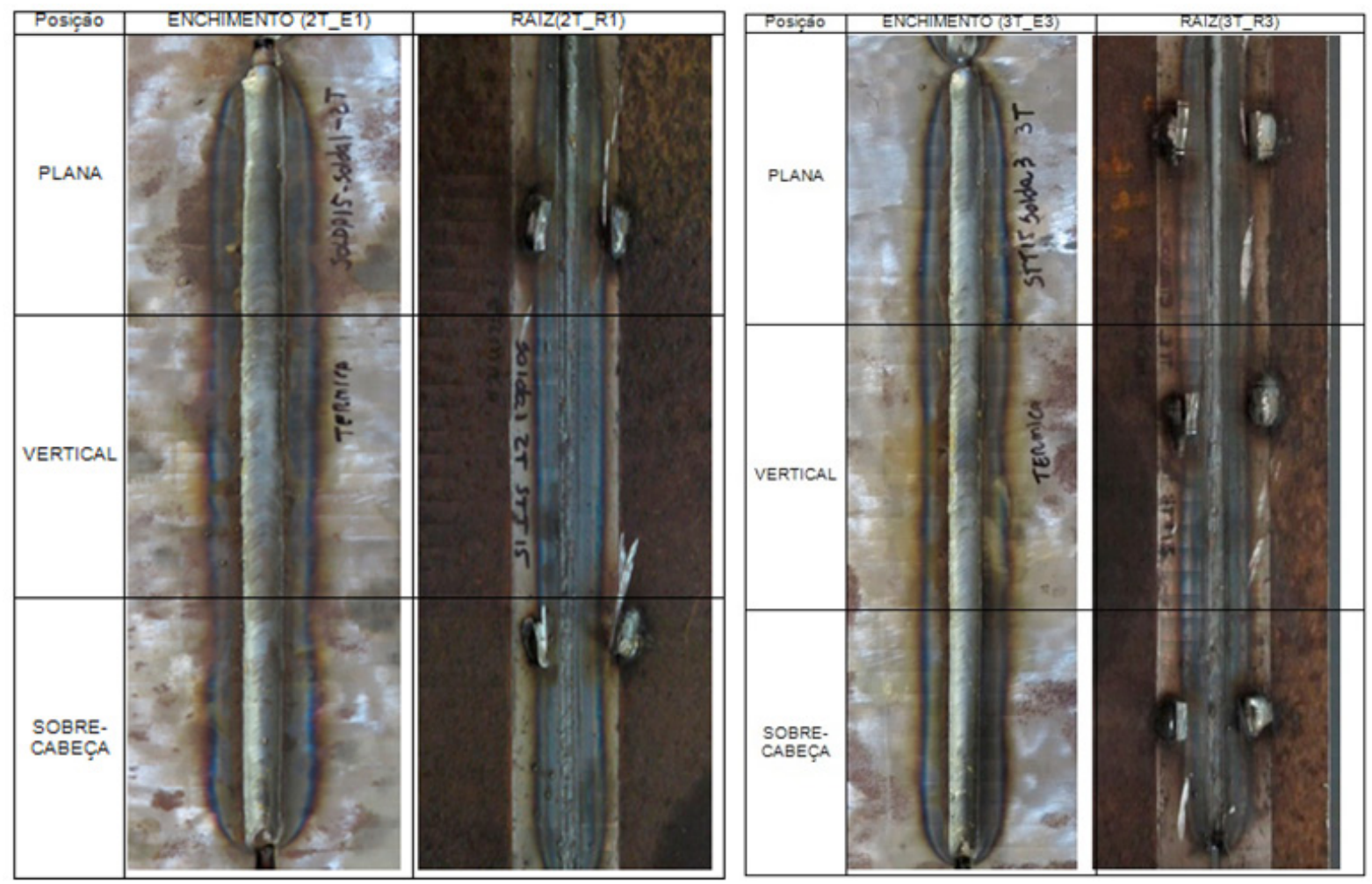

Figura 12. Cordões de solda típico fabricado em testes com câmera termográfica².

Todos os gráficos (temperatura x tempo) examinados para os passes de enchimento e de raiz são mostrados na Figura 13. Cada gráfico está identificado conforme as juntas soldadas/ensaios apresentadas na Tabela 5.

Para melhor entendimento dos gráficos apresentados na Figura 13, os autores chamam a atenção do leitor da necessidade de confrontar a Figura 7, a Tabela 5 e os pontos apresentados no canto inferior esquerdo de cada gráfico para cada ensaio. Após esta verificação, você leitor, chegará a conclusão que o primeiro gráfico superior representa o centro do cordão de solda e os gráficos inferiores de cada ensaio/junta soldada evidencia pontos simétricos mais distantes em relação a este centro. Logo, o primeiro gráfico inferior de cada ensaio retrata o ponto simétrico mais distante da superfície do corpo de prova analisado.

Assim sendo, é possível verificar que pontos próximos ao cordão de solda estão em processo de esfriamento e pontos mais distantes estão em processo de aquecimento, manifestando assim a propagação do calor.

\footnotetext{
2 Apesar dos cordões de solda serem circulares (meia cana de tubulação) as imagens serão apresentadas no formato de imagens planificadas concentrando as três posições de soldagem em uma única imagem. A técnica de planificação aqui aplicada é descrita detalhadamente em Nascimento (2015) (autor da técnica).
} 

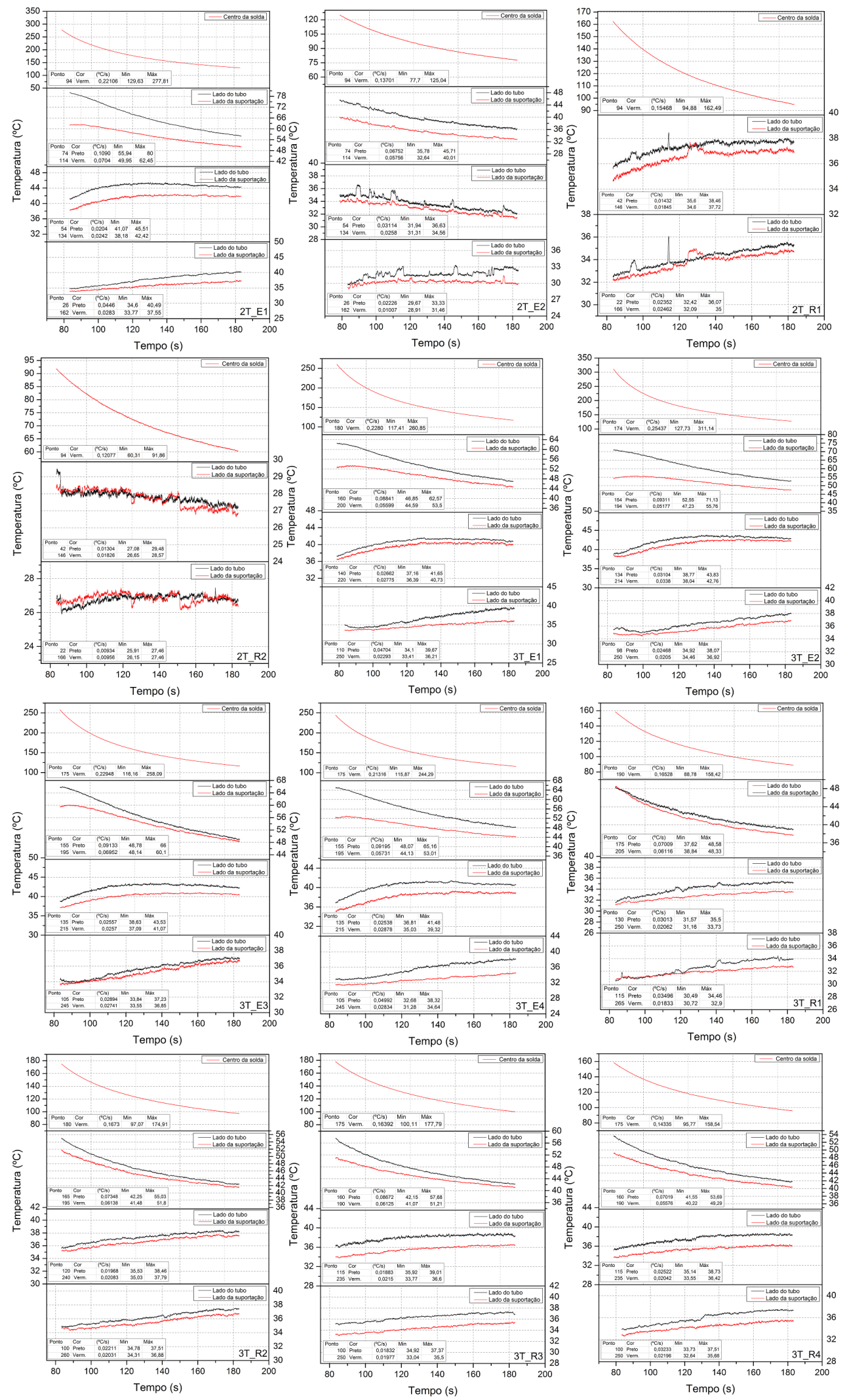

Figura 13. Gráficos (temperatura x tempo) do centro da solda (gráfico com uma única curva) e nos pontos simétricos para o passe de raiz e de enchimento (linhas de cor vermelha: lado da suportação; linhas cor preta: lado do tubo) para cada ensaio/junta soldada apresentada na Tabela 5. 
Os resultados mostram também que em todos os pontos simétricos examinados, tanto para o passe de raiz quanto para o passe de enchimento, os pontos localizados do lado da suportação, apresentam-se com temperaturas mais baixas em relação aos pontos localizados do lado do tubo. Isto mostra que a difusividade térmica $(\alpha)$ do lado da suportação ou o escoamento do fluxo de calor é maior do que a do lado do tubo, ou seja, em consequência da grande quantidade de massa (ver Figura 4) que contribui para a propagação por condução do calor. Este trabalho sugere que essa diferença pode ser contida utilizando tecimento com parada lateral durante as soldagens.

Observa-se também que a diferença de temperatura entre ambos os lados avaliados é de $4{ }^{\circ} \mathrm{C}$ na pior das situações (diferença entre as curvas de cor preta e vermelha). Já a utilização de 3 tubos ou 2 tubos na montagem do duto não interferiu significativamente nos resultados.

Outro fato que demonstra a robustez do sistema é a coloração uniforme e simétrica dos óxidos formados pelo aquecimento em ambos os lados do cordão de solda, como pode ser observada na Figura 12.

Por fim, fica evidenciada a importância do estudo supracitado, pois, tais fenômenos poderão ocorrer como, por exemplo, na união de flanges em dutos ou tubulações, em juntas que apresentam espessuras diferentes entre outros.

\section{Conclusões}

É possível afirmar que a utilização de montagens com duas ou três seções de tubos de teste não interferiu significantemente nos resultados e que a diferença de temperatura de $4^{\circ} \mathrm{C}$, na pior das situações entre ambos os lados do duto para pontos simétricos, não é significativa para processos de soldagens. Assim, a diferença de massa existente em ambos os lados da instalação, não contribuem para a falta de fusão lateral frequente encontrada por Vilarinho et al. (2014), podendo ser contornado com um conjunto robusto de parâmetros.

\section{Agradecimentos}

Os autores agradecem à CAPES, ao CNPq e à FAPEMIG pelas bolsas de estudo e pesquisa aos autores. Agradecem à PETROBRAS, pela doação dos consumíveis e incentivos a pesquisa e, aos membros e colaboradores do Laprosolda/UFU (Grupo Centro para Pesquisa e Desenvolvimento de Processos de Soldagem) pelo apoio e frutíferas discussões. Enfim, agradecem ao Programa de Pós-graduação da Universidade Federal de Uberlândia (COPEM), pela oportunidade.

\section{Referências}

American Petroleum Institute. API 5L: specification for line pipe. 45th ed. Washington: API; 2012. 180 p.

FLIR System. ThermoVision ${ }^{\text {TM }}$ SDK. Versão 2.6: user's manual. Kyffhäuserland: FLIR; 2009.

FLIR System. ThermoVision ${ }^{\mathrm{TM}}$ LabView $^{\circledR}$ Toolkit: user's manual. Kyffhäuserland: FLIR; 2010 [acesso em 20 jun. 2016]. Disponível em: http://www.thermokameras.com/ir-systeme/software/ Handbuch_ResearchIR.pdf

International Organization for Standardization. ISO 17662: welding: calibration, verification and validation of equipamento used for welding, including ancillary activities. Genebra: ISO; 2005. 38 p.

Lincoln Electric. Technology gets to the root of pipe welding [página da internet]. USA: Lincoln Electric; 2014 [acesso em 3 abr. 2014]. Disponível em: www.lincolneletric.com
Machado MVR. Sistema embarcado sem fio para monitoramento de sinais em soldagem a arco elétrico com abordagem tecnológica [dissertação de mestrado]. Uberlândia: Universidade Federal de Uberlândia; 2011.

Mota CP. Sistema de visão por infravermelho próximo para monitoramento de processos de soldagem a arco [dissertação de mestrado]. Uberlândia: Universidade Federal de Uberlândia; 2011.

Nascimento LA. Avaliação dos processos GMAW com transferência por curto-circuito convencional e controlado (STT) aplicados na soldagem circunferencial mecanizada [dissertação de mestrado]. Uberlândia: Universidade Federal de Uberlândia; 2015. p. 72-285.

Vilarinho LO, Nascimento LA, Acuna AF, Carvalho LP, Freitas JC. Conventional and controlled short-circuit GMAW processes for downhill pipe welding. In: FABTECH Show 2014; 2014; Atlanta, Georgia. Atlanta: FABTECH; 2014. p. 289-295. 